\title{
Focal Leptomeningeal Disease with Perivascular Invasion in EGFR-Mutant Non-Small-Cell Lung Cancer
}

\author{
(D) A. Dasgupta, (D)F.Y. Moraes, (D) R. Rawal, (DP. Diamandis, and (D)D.B. Shultz
}

\begin{abstract}
SUMMARY: We report a previously undescribed pattern of brain metastases in patients with epidermal growth factor receptormutated non-small-cell lung cancer treated with tyrosine kinase inhibitors and radiation therapy. These highly distinct lesions appear to spread focally within the leptomeninges, with invasion along the perivascular spaces (FLIP). The survival of patients with FLIP was significantly better compared with patients with classic leptomeningeal disease (median survival, 21 versus 3 months; $P=$.003). It is unclear whether this pattern of growth is unique to epidermal growth factor receptor-mutated non-small-cell lung cancer.
\end{abstract}

ABBREVIATIONS: EGFRm = epidermal growth factor receptor-mutated; $\mathrm{CLMD}=$ classic leptomeningeal disease; FLIP $=$ focal leptomeningeal involvement with invasion of the perivascular space; NSCLC = non-small-cell lung cancer; TKI = tyrosine kinase inhibitors; WBRT = whole-brain radiation

W e report a previously undescribed pattern of brain metastases in epidermal growth factor receptor-mutated (EGFRm) non-small-cell lung cancer (NSCLC) that appears to spread focally within the leptomeninges, with invasion along the perivascular spaces (FLIP). FLIP is radiologically and clinically distinct from classic leptomeningeal disease (cLMD). We present 5 cases.

\section{Case Series}

Patient 1. A 67-year-old woman with locally advanced EGFRm NSCLC (exon 19 mutation) was treated with lobectomy, mediastinal node dissection, and 4 cycles of adjuvant cisplatin and vinorelbine. Eighteen months later she relapsed in the mediastinum. A restaging brain MR imaging showed a ring-enhancing lesion associated with leptomeningeal changes in the right parietal lobe (Fig 1). She declined brain radiation therapy or surgery and began treatment with afatinib. Three months later her brain lesion progressed radiographically, though she remained asymptomatic, and a decision was made to proceed with surgical resection. Pathologic analysis revealed brain tissue invaded by a metastatic adenocarcinoma growing in sheets and papillary structures. The tumor cells exhibited nuclear atypia and readily

Received March 30, 2020; accepted after revision May 7.

From the Department of Radiation Oncology (A.D., F.Y.M., D.B.S.), Division of Neuroimaging, Joint Department of Medical Imaging (S.R.), and Department of Neuropathology (P.D.), Princess Margaret Cancer Centre and University Health Network, Toronto, Ontario, Canada.

Please address correspondence to David B. Shultz, MD, Department of Radiation Oncology, Princess Margaret Cancer Centre, University of Toronto, 7th Floor of Ontario Power Generation (OPG) Building, Room 7-401, 700 University Ave, Toronto, ON M5G 2M9, Canada; e-mail: david.shultz@rmp.uhn.ca

http://dx.doi.org/10.3174/ajnr.A6640 identifiable mitoses and had an immunohistochemical pattern (CK7+, CK20-, TTF1+) supportive of lung origin. There were many examples in which the tumor showed growth and spread along the perivascular and leptomeningeal spaces. Given this finding of focal leptomeningeal involvement with invasion of the perivascular space, we henceforth refer to this pattern as FLIP (Fig 2). She was treated with whole-brain radiation (WBRT) and continued afatinib. Thereafter, she remained clinically and radiographically stable for 12 months, at which time she developed widespread leptomeningeal disease. She died 6 months later.

Patient 2. A 62-year-old man with early-stage EGFRm NSCLC (exon 19 deletion) was initially treated with a left lower lobectomy. Thirty-four months later, he developed pulmonary metastases and began treatment with gefitinib. Twelve months after that, he developed headaches, and a brain MR imaging revealed a lesion with an appearance consistent with FLIP in the right cerebellum (Fig 3) and several additional small lesions in the brain parenchyma. He was treated with WBRT and continued gefitinib. An MR imaging 3 months later revealed a complete response in his other brain lesions and a partial response in the FLIP. Unfortunately, MR imaging 4 months later showed isolated progression of the FLIP, with extension along the cerebellar folia and minimal infiltration into the brain parenchyma. He remained asymptomatic. The FLIP lesion was targeted with focal radiation therapy, and 3 months later, MR imaging showed stable disease. Unfortunately, MR imaging performed 4 months later demonstrated radiologic progression of the FLIP, which continued for 12 months until he died due to neurologic causes. Throughout 
this, no additional brain lesions were noted, and his extracranial disease remained stable.

Patient 3. A 49-year-old man with early-stage EGFRm NSCLC (exon 20 mutation) was treated with lobectomy. Eighteen months later he developed right-sided hemiparesis, and a brain MR

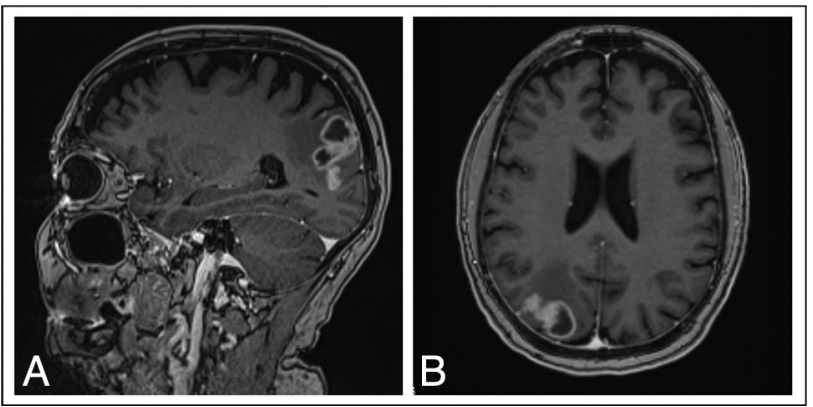

FIG 1. Brain MR imaging for patient 1, a 67-year-old woman with locally advanced EGFRm NSCLC. Sagital (A) and axial (B) projections show enhancement along the parenchyma, with ring-enhancing lesions extending into the right parietal sulci in continuity. The scan was performed for restaging purposes, before any brain-directed treatment. imaging revealed 3 parenchymal metastases, including one in the left parietal lobe. He was treated with WBRT followed by carboplatin and pemetrexed, and his hemiparesis resolved. Fourteen months later, a lesion in the left parietal lobe progressed consistent with FLIP (Fig 4). The lesion was treated with salvage stereotactic radiosurgery; however, an MR imaging 3 months later showed progression. During that same period, he progressed extracranially and began treatment with pembrolizumab. Unfortunately, follow-up MR imaging 6 months later showed continued progression of the FLIP, though the rest of his brain remained stable. He was given the option of surgery, which he declined, and he died 2 months later.

Patient 4. A 77-year-old woman presented with multiple lung nodules, bone metastases, and 2 small brain metastases, one in the cerebellum and the other in the right frontal lobe. A lung biopsy revealed EGFRm NSCLC (L858R mutation), and she was treated with radiation for her primary lung mass and gefitinib. While initially MR imaging revealed a partial response in her brain to gefitinib, 18 months later the lesion in the right frontal lobe progressed with an appearance consistent with FLIP (Fig 5). The patient was asymptomatic and was treated with WBRT. Following that, brain MRIs performed up to 7 months later revealed marked improvement of the FLIP and stable disease elsewhere, and she remained symptom-free. Ultimately, her systemic disease progressed, and she died 8 months following WBRT.

Patient 5. A 46-year-old woman was diagnosed with locally advanced EGFRm NSCLC (exon19 mutation) and underwent lobectomy and lymph node dissection followed by adjuvant chemotherapy and thoracic radiation therapy. Brain MR imaging at that time revealed no evidence of intracranial disease. One year later, she developed lung metastases and began gefitinib. Again, an MR imaging of the brain was performed and was unremarkable. After 4 years of gefitinib, she developed headaches and was found to have 3 brain metastases treated with salvage stereotactic radiosurgery, including one in the right cerebellum. All lesions initially responded. One year later, the patient developed mild headaches, and MR imaging revealed progression of the right cerebellar lesion consistent with FLIP (Fig 6). She underwent resection, revealing adenocarcinoma growing in a papillary pattern with large areas of necrosis and focal leptomeningeal involvement. Unfortunately, although there were areas suspicious for perivascular space invasion, the
FIG 2. Histopathology. A, Hematoxylin-eosin image shows cohesive epithelioid neoplastic cells closely associated with parenchymal blood vessels (black arrows). Immunohistochemical staining (brown coloration) for CK7 (B) and TTFl (C) supports a lung origin and highlights the involvement of the perivascular spaces. $D$, Growth of tumor within the Virchow-Robins spaces (marked brown again with CK7 immunostaining) creates an impression of scattered tumor foci within the unstained brain tissue rather than the typical solitary metastatic mass. $E$ and $F$, Evidence of leptomeningeal involvement with adenocarcinoma closely associated with and encircling larger thick meningeal-type vessels (black arrows). F, A high-power view of the red box seen in $E$ highlights that most of the increased cellularity is tumor cells. 


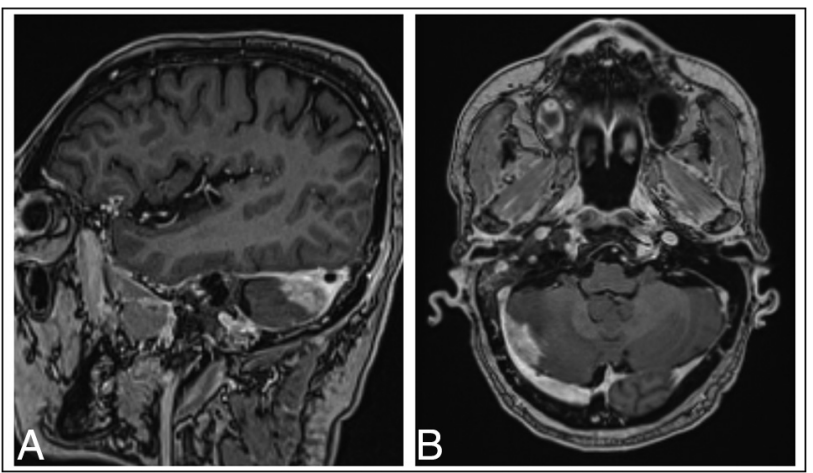

FIG 3. Brain MR imaging for patient 2, a 62-year-old man with EGFRm NSCLC. Sagital $(A)$ and axial $(B)$ projections demonstrate an enhancing lesion spreading along the cerebellar folia after several months of treatment with gefitinib.
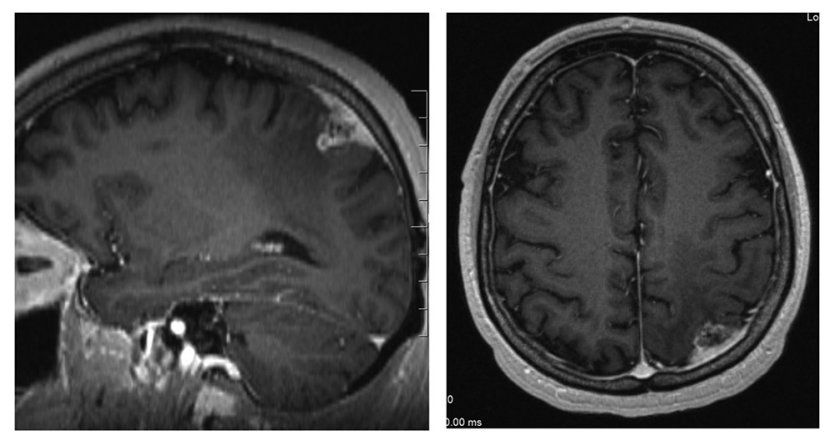

FIG 4. Brain MR imaging for patient 3, a 49-year-old man with EGFRm NSCLC with an enhancing lesion in the left parietal lobe 14 months following WBRT.

specimen was too necrotic for definitive characterization of that. Six months after that, the lesion progressed again and was treated with fractionated radiation. Unfortunately, the tumor recurred 6 months later, requiring a second resection. Three months following that, her brain MR imaging showed stable disease; however, symptomatically, she was severely fatigued, without an obvious cause, and 6 months later she died in the setting of palliative care. Throughout this time, since her initial radiation therapy, the remainder of the patient's brain showed no evidence of additional tumors. At no point did she have focal neurologic findings or symptomatology suggestive of widespread leptomeningeal disease. Her extracranial disease remained well-controlled throughout the course of her illness.

Comparison with CLMD. In a previously described clinical data base of 198 patients with central nervous system involvement from EGFRm NSCLC, ${ }^{1}$ we identified 12 patients with CLMD and compared their survival with that of patients with FLIP using a KaplanMeier analysis. Overall survival was estimated from the first radiologic evidence of leptomeningeal disease or FLIP until death. We used the log-rank test to compare the 2 groups. In this analysis, survival following FLIP was significantly improved compared with cLMD (median, 21 versus 3 months; $P=.003$ ) (Fig 7). The survival of our cLMD group was similar to that of a cohort of 212 patients

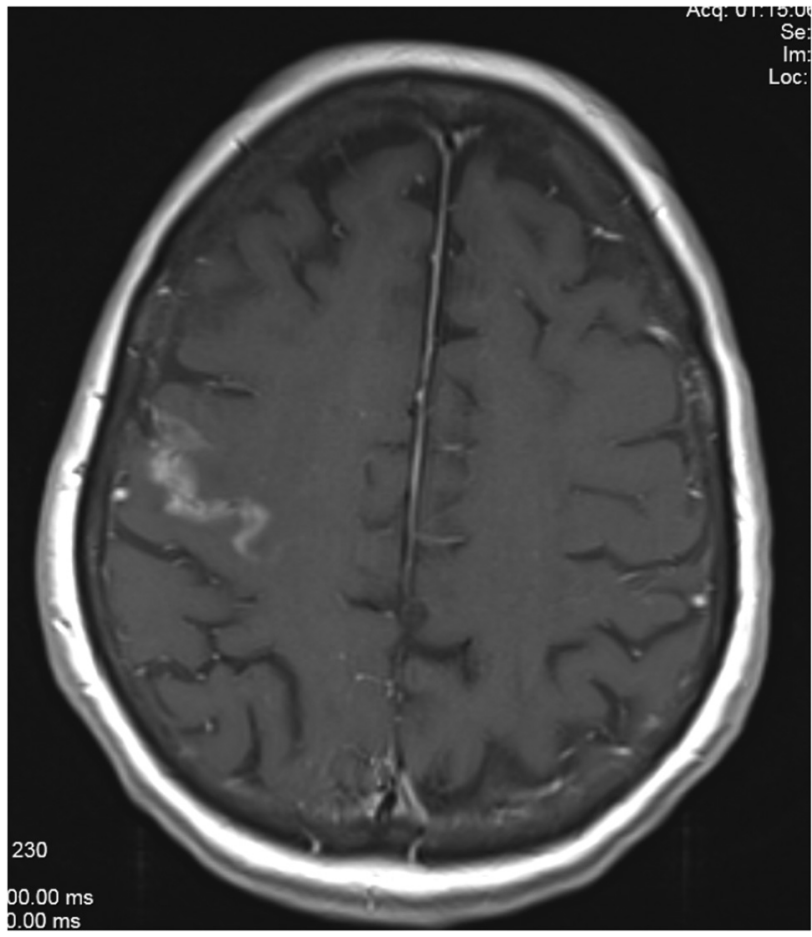

FIG 5. Brain MR imaging for patient 4, a 77-year-old woman with EGFRm NSCLC. Axial projection demonstrates a right frontal lobe lesion following 18 months of gefitinib treatment.
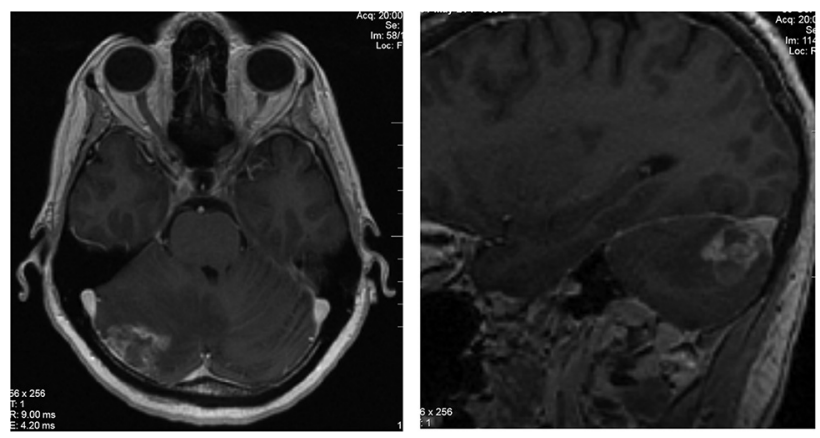

FIG 6. Brain MR imaging for patient 5, a 46-year-old woman with EGFRm NSCLC. Sagital $(A)$ and axial $(B)$ projections demonstrate an enhancing right cerebellar lesion with spread along the cerebellar folia, 1year following stereotactic radiosurgery and several years of gefitinib treatment.

with NSCLC with CLMD (including 75 EGFRm), in which the median survival was 4.5 months. ${ }^{2}$

\section{DISCUSSION}

Herein we describe a unique pattern of intracranial metastasis termed FLIP. Prior reports have described lesions consistent with limited leptomeningeal involvement that while focal, otherwise bore a strong radiographic resemblance to classic leptomeningeal disease. ${ }^{3}$ FLIP is distinct from that entity radiographically. It is also highly distinct from widespread or focal pachymeningeal failures that sometimes arise following postoperative salvage stereotactic radiosurgery. ${ }^{4}$ 


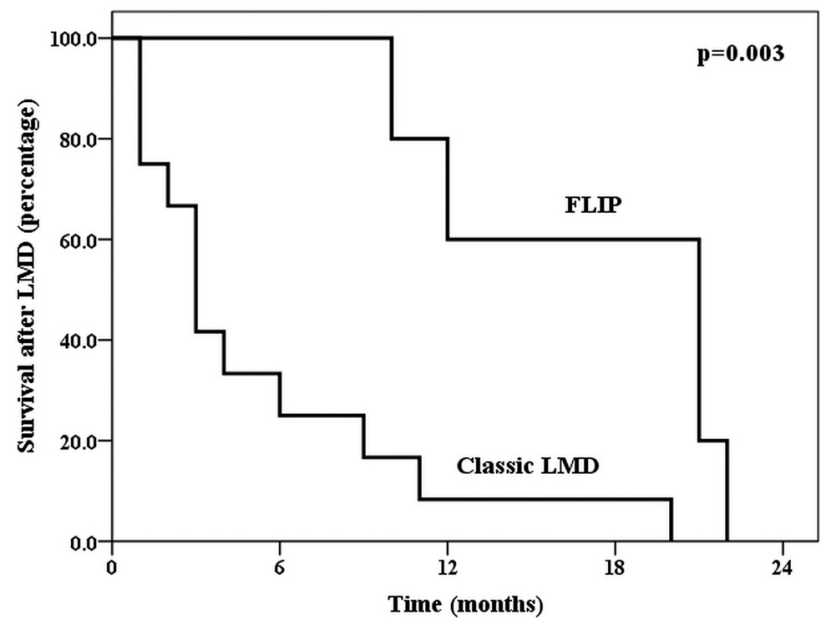

FIG 7. Kaplan-Meier survival plot comparing survival outcomes of FLIP with those of classic leptomeningeal disease in EGFRm NSCLC.

Previous studies have described features associated with cLMD from EGFRm NSCLC. ${ }^{5,6}$ In a series of 144 patients with EGFRm NSCLC (15 had cLMD), Lin et $\mathrm{al}^{5}$ observed that some tyrosine kinase inhibitors (TKIs) exerted improved CNS control compared with others, including in patients with cLMD. Likewise, in a case series of 5 patients with EGFRm NSCLC undergoing treatment with a TKI, Sener et $\mathrm{al}^{6}$ noted that clinical signs and symptoms of cLMD preceded radiographic findings by several months, suggesting that TKIs may mask occult leptomeningeal involvement. Both studies highlight the interplay between TKI activity and disease progression within the leptomeninges in EGFRm NSCLC and how that may affect clinical and radiographic presentation. In a recently published Phase I study of 41 patients with CLMD, Yang et $\mathrm{al}^{7}$ reported a median overall survival of 11 months with the use of a third-generation TKI (osimertinib). With newer generations of TKI with better CNS penetration, the survival of patients with cLMD is expected to improve. From our own limited study, it is unclear whether FLIP reflects unique biologic features of EGFRm NSCLC brain metastases or a response to $E G F R$-targeting agents.

Perivascular invasion from metastatic carcinoma has been previously reported. ${ }^{8,9}$ Kleinschmidt-DeMasters and Damek $^{8}$ reported 2 such cases, both in patients with NSCLC (molecular status not available) who had previous bevacizumab treatment. Both patients presented with severe neurologic symptoms, including dizziness and cognitive decline, and similar findings on MR imaging (deep white matter hyperintensities and disseminated leptomeningeal disease). At postmortem examination of 1 patient, pathology revealed perivascular involvement. Takei et al ${ }^{9}$ reported extensive intravascular carcinomatosis in the central nervous system of a patient who succumbed to inflammatory metastatic breast cancer. Clinically and radiologically, however, these examples were dissimilar to FLIP, which appears to be a focal process, at least radiographically, and is associated with mild symptomatology. Our analysis also indicates that survival following a diagnosis of FLIP may be superior to that in CLMD, notwithstanding the inherent potential for bias when comparing 2 retrospectively identified cohorts.
The natural history of some of the cases of FLIP presented herein suggests growth from a parenchymal lesion to the leptomeninges, rather than seeding through the CSF, with spread along perivascular spaces with perivascular channels acting as a bridge between the brain parenchyma and the leptomeningeal surface, ${ }^{10}$ similar to processes referred to as vascular co-option or angiotropism. ${ }^{11,12}$ Three patients in our series had associated dural thickening and enhancement, which may represent a combination of tumor involvement and venous congestion. Of note, none of the patients in our series underwent cytologic analysis of CSF. Also, contrast-enhanced MR imaging of the spine was not available for any of the patients following the development of FLIP. To further investigate this entity, we plan to characterize this pattern of failure in a larger cohort.

Disclosures: Phedias Diamandis—UNRELATED: Employment: University Health Network.

\section{REFERENCES}

1. Ramotar M, Barnes S, Moraes F, et al. Neurological death is common in patients with EGFR mutant non-small cell lung cancer diagnosed with brain metastases. Adv Radiat Oncol 2020;5:350-57 CrossRef

2. Liao BC, Lee JH, Lin CC, et al. Epidermal growth factor receptor tyrosine kinase inhibitors for non-small-cell lung cancer patients with leptomeningeal carcinomatosis. J Thorac Oncol 2015;10:1754-61 CrossRef Medline

3. Wolf A, Donahue B, Silverman JS, et al. Stereotactic radiosurgery for focal leptomeningeal disease in patients with brain metastases. J Neurooncol 2017;134:139-43 CrossRef Medline

4. Cagney DN, Lamba N, Sinha S, et al. Association of neurosurgical resection with development of pachymeningeal seeding in patients with brain metastases. JAMA Oncol 2019;5:703-09 CrossRef

5. Lin CY, Chang CC, Su PL, et al. Brain MRI imaging characteristics predict treatment response and outcome in patients with de novo brain metastasis of EGFR-mutated NSCLC. Medicine (Baltimore) 2019;98:e16766 CrossRef Medline

6. Sener U, Matin N, Yu H, et al. Radiographic appearance of leptomeningeal disease in patients with EGFR-mutated non-small-cell lung carcinoma treated with tyrosine kinase inhibitors: a case series. CNS Oncol 2019;8:CNS4 CrossRef Medline

7. Yang JC, Kim SW, Kim DW, et al. Osimertinib in patients with epidermal growth factor receptor mutation-positive non-small-cell lung cancer and leptomeningeal metastases: the BLOOM study. $J$ Clin Oncol 2020;38:538-47 CrossRef Medline

8. Kleinschmidt-DeMasters BK, Damek DM. The imaging and neuropathological effects of bevacizumab (Avastin) in patients with leptomeningeal carcinomatosis. J Neurooncol 2010;96:375-84 CrossRef Medline

9. Takei H, Rouah E, Barrios R. Intravascular carcinomatosis of central nervous system due to metastatic inflammatory breast cancer: a case report. Neuropathology 2015;35:456-61 CrossRef Medline

10. Groeschel S, Chong WK, Surtees R, et al. Virchow-Robin spaces on magnetic resonance images: normative data, their dilatation, and a review of the literature. Neuroradiology 2006;48:745-54 CrossRef Medline

11. Valiente M, Obenauf AC, Jin X, et al. Serpins promote cancer cell survival and vascular co-option in brain metastasis. Cell 2014;156:1002-16 CrossRef Medline

12. Bentolila LA, Prakash R, Mihic-Probst D, et al. Imaging of angiotropism/vascular co-option in a murine model of brain melanoma: implications for melanoma progression along extravascular pathways. Sci Rep 2016;6:23834 CrossRef Medline 